Idiopathische pulmonale Fibrose

\title{
Hohe akute Mortalität nach Exazerbationen
}

Song JW et al. Acute exacerbation of idiopathic pulmonary fibrosis: incidence, risk factors and outcome. Eur Respir J. 2010 (E-pub ahead of print)

\section{Fragestellung}

Die vorliegende Studie untersuchte bei Patienten mit idiopathischer pulmonaler Fibrose (IPF) die Inzidenz, die Risikofaktoren und die prognostischen Konsequenzen von akuten klinischen Verschlechterungen, die zu einer Hospitalisierung geführt haben, unabhängig von deren Ursache. Ein besonderer Fokus lag auf sogenannten akuten Exazerbationen der IPF. Hierunter versteht man eine ungeklärte Verschlechterung oder ein Auftreten von Dyspnoe innerhalb von 30 Tagen zusammen mit neu aufgetretenen bilateralen Milchglastrübungen und/oder Konsolidierungen auf der Basis bereits existenter mit einem Muster der gewöhnlichen interstitiellen Pneumonie (usual interstitial pneumonia, UIP) zu vereinbarenden Veränderungen („Honigwaben“, retikuläre Veränderungen) in der hoch auflösenden Computertomografie (HR-CT). Dabei sind bekannte Ursachen einer akuten Verschlechterung, insbesondere pulmonale Infekte, aber auch eine Lungenembolie und Linksherzversagen auszuschließen [1].

\section{Methodik}

Es handelt sich um eine retrospektive Datenanalyse von 461 IPF-Patienten, von denen 269 eine histologisch gesicherte Diagnose hatten. Im Median wurden die Patienten für 22,9 Monate beobachtet.

\section{Ergebnisse}

Bei 163 Patienten (35,4\%) kam es zu mindestens einem Ereignis einer akuten klinischen Verschlechterung mit einer Hospitalisierung. 42 Patienten erlitten mehrere derartige Ereignisse.

Häufigste Ursachen für ein derartiges Ereignis waren akute Exazerbationen entsprechend der o.g. Definition (90 Patienten; 55,2\% der Ereignisse) und
Infektionen (51 Patienten; 31,3\%), wobei letztere vor allem bei Patienten, die mit Steroiden behandelt wurden, eine Rolle spielten. Weitere Ursachen waren ein Pneumothorax $(n=9 ; 2,0 \%)$, eine kardiale Dekompensation $(\mathrm{n}=5 ; 1,1 \%)$ und Lungenembolien $(\mathrm{n}=2 ; 0,4 \%)$. Die 1- bzw. 3-Jahres-Inzidenzen für akute Exazerbationen betrugen 14,2 bzw. 20,7\%.

Riskofaktoren für akute Exazerbationen waren eine fortgeschrittene Einschränkung der Lungenfunktion (niedrige forcierte Vitalkapazität [FVC], Diffusionskapazität für Kohlenmonoxid [DL $\mathrm{DO}_{\mathrm{CO}}$ und totale Lungenkapazität [TLC]) und eine negative Raucheranamnese. Bei 16 Patienten ging der akuten Exazerbation eine Intervention, z.B. eine videoassistierte Thorakoskopie, voraus.

50\% der Patienten mit einem Ereignis einer akuten klinischen Verschlechterung mit einer Hospitalisierung starben noch im Krankenhaus. Die mediane Überlebenszeit war 2,2 Monate. Diese schlechte Prognose war unabhängig davon, ob dem Ereignis ein Infekt oder eine akute Exazerbation zugrunde lag.

Derartige Ereignisse wirkten sich auch auf die Langzeitprognose negativ aus (ab Diagnosestellung der Grunderkrankung). Bei Patienten mit akuten Exazerbationen war die mediane Überlebenszeit nach der Diagnosestellung signifikant kürzer als bei Patienten ohne derartige Ereignisse (15,5 vs. 60,6 Monate; $\mathrm{p}<0,001)$. Die entsprechenden 5-Jahres-Überlebensraten betrugen 18,4 und 50,0\% $(p<0,001)$.

Neben dem Auftreten von akuten Exazerbationen erwiesen sich ein höheres Lebensalter, eine schlechte Lungenfunktion und eine Behandlung mit Steroiden und/oder zytotoxischen Substanzen als unabhängige Prädiktoren für eine schlechte Prognose.

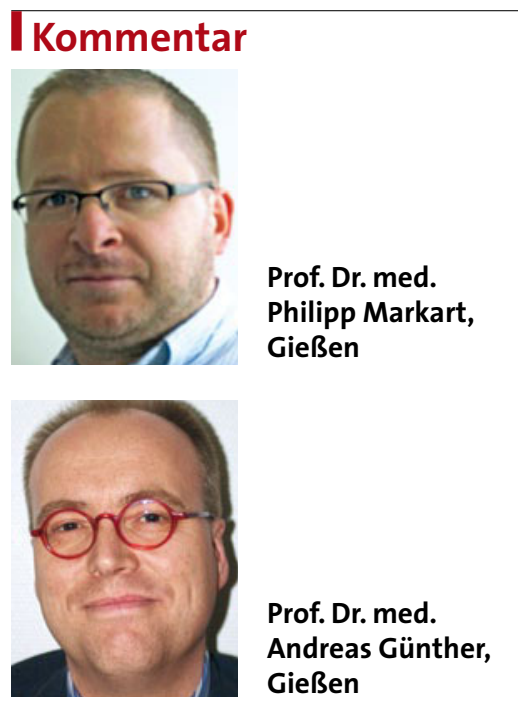

Die wesentlichen Erkenntnisse dieser Studie sind: Akute, zur Krankenhauseinweisung führende Verschlechterungen treten bei IPF-Patienten häufig auf, sie sind mit einer hohen akuten Letalität verbunden und wirken sich negativ auf die Langzeitprognose aus. Als Hauptursachen derartiger Ereignisse wurden akute Exazerbationen und Infektionen identifiziert.

Erfahrungsgemäß ist der Nachweis einer Infektion bzw. die Abgrenzung einer Infektion von einer akuten Exazerbation, bei der entsprechend der o.g. Definition eine Infektion ausgeschlossen sein soll, oft schwierig. Daher ist es durchaus denkbar, dass der Anteil der Infektionen unterschätzt wurde. Immerhin wurde bei 52,8\% der Patienten zum Keimnachweis bzw -ausschluss eine bronchoalveoläre Lavage bzw. eine Untersuchung des Trachealsekrets durchgeführt. Effektive Therapieoptionen für akute Exazerbationen liegen derzeit nicht vor.

Insbesondere eine fortgeschrittene Erkrankung scheint für derartige Ereignisse zu prädisponieren. Darüber hinaus wurde eine Behandlung mit Steroiden als ein wesentlicher Risikofaktor für infektgetriggerte akute Verschlechterungen identifiziert. Außerdem erwies sich eine Behandlung mit Steroiden und/oder zytotoxischen Substanzen als ein unabhängiger Prädiktor für eine schlechte Prognose bei IPF-Patienten. Dies unterstreicht erneut die Notwendigkeit des restriktiven Einsatzes dieser Substanzen bei IPF-Patienten.

\footnotetext{
Literatur

1. Collard et al. Am J Respir Crit Care Med. 2007;176: $636-643$
} 